OPEN ACCESS

Edited by: László Vécsei,

University of Szeged, Hungary

Reviewed by: Abed N. Azab, Ben-Gurion University of the Negev.

Israel

Eva Z. Reininghaus, Medical University of Graz, Austria

${ }^{*}$ Correspondence: Kaat Hebbrecht kaat.hebbrecht@emmaus.be

Specialty section: This article was submitted to Inflammation,

a section of the journal

Frontiers in Immunology

Received: 12 February 2021 Accepted: 13 April 2021

Published: 19 May 2021

Citation: Hebbrecht K, Skorobogatov K, Giltay EJ, Coppens V, De Picker L and Morrens M (2021) Tryptophan Catabolites in Bipolar Disorder: A Meta-Analysis.

Front. Immunol. 12:667179. doi: 10.3389/fimmu.2021.667179

\section{Tryptophan Catabolites in Bipolar Disorder: A Meta-Analysis}

\author{
Kaat Hebbrecht ${ }^{1,2 *}$, Katrien Skorobogatov ${ }^{1,2}$, Erik J. Giltay ${ }^{1,3}$, Violette Coppens ${ }^{1,2}$, \\ Livia De Picker ${ }^{1,2}$ and Manuel Morrens ${ }^{1,2}$ \\ 1 Faculty of Medicine and Health Sciences, Collaborative Antwerp Psychiatric Research Institute (CAPRI), University of \\ Antwerp, Antwerp, Belgium, ${ }^{2}$ Scientific Initiative of Neuropsychiatric and Psychopharmacological Studies (SINAPS), \\ University Psychiatric Centre Duffel, Duffel, Belgium, ${ }^{3}$ Department of Psychiatry, Leiden University Medical Center, \\ Leiden, Netherlands
}

Objective: Tryptophan catabolites (TRYCATs) are implicated in the pathophysiology of mood disorders by mediating immune-inflammation and neurodegenerative processes. We performed a meta-analysis of TRYCAT levels in bipolar disorder (BD) patients compared to healthy controls.

Methods: A systematic literature search in seven electronic databases (PubMed, Embase, Web of Science, Cochrane, Emcare, PsycINFO, Academic Search Premier) was conducted on TRYCAT levels in cerebrospinal fluid or peripheral blood according to the PRISMA statement. A minimum of three studies per TRYCAT was required for inclusion. Standardized mean differences (SMD) were computed using random effect models. Subgroup analyses were performed for BD patients in a different mood state (depressed, manic). The methodological quality of the studies was rated using the modified Newcastle-Ottawa Quality assessment Scale.

Results: Twenty-one eligible studies were identified. Peripheral levels of tryptophan (SMD = $-0.44 ; p<0.001)$, kynurenine $(\mathrm{SMD}=-0.3 ; p=0.001)$ and kynurenic acid $(\mathrm{SMD}=-.45$; $p=<0.001$ ) were lower in BD patients versus healthy controls. In the only three eligible studies investigating TRP in cerebrospinal fluid, tryptophan was not significantly different between BD and healthy controls. The methodological quality of the studies was moderate. Subgroup analyses revealed no significant difference in TRP and KYN values between manic and depressed BD patients, but these results were based on a limited number of studies.

Conclusion: The TRYCAT pathway appears to be downregulated in BD patients. There is a need for more and high-quality studies of peripheral and central TRYCAT levels, preferably using longitudinal designs.

Keywords: bipolar disorder, inflammation, immune, kynurenine, tryptophan, depression 


\section{INTRODUCTION}

Bipolar disorder (BD) is a chronic psychiatric disorder characterized by alternating periods of depression and abnormally elevated moods. BD is one of the leading causes of global disability, resulting in cognitive and functional decline and an increased mortality rate (1). The pathophysiology of BD remains to be fully elucidated but accumulating evidence points towards a pathophysiological role of chronic low-grade inflammation (2).

The kynurenine pathway of tryptophan (TRP) degradation has been proposed as the missing link through which inflammation causes neurotoxicity and psychiatric symptoms. TRP is an essential amino acid and a precursor for serotonin or 5-hydroxytryptamine. In response to inflammation or psychosocial stress (3), TRP is primarily metabolized into kynurenine $(\mathrm{KYN})$ following an upregulation of indoleamine 2,3-deoxygenase (IDO-1) and hereby leading to a reduction in availability of serotonin (for a graphical illustration of the KYN Pathway, see Figure 1). This depletion of serotonin has been assumed to play a major role in the pathophysiology of depression $(5,6)$. More recent studies also point towards the imbalance supposedly neurotoxic [including 3-hydroxy kynurenine (3-HK) and quinolinic acid (QA)) and neuroprotective (kynurenic acid (KA)] TRP catabolites (TRYCAT) as a central mechanism in the pathophysiology of mood disorders $(7,8)$. In patients with Major Depressive Disorder (MDD), a consistent increase in 3-HK and QA and a decrease in KA in blood and cerebrospinal fluid has been found $(8,9)$. In BD patients, however, results have been more divergent and appear specific to the symptomatic state (10). In depressed or euthymic BD patients, TRYCAT alterations seem to be similar to those in MDD (11-13). In contrast, BD patients with a history of psychosis have shown elevated KA levels in cerebrospinal fluid (CSF) but not in the periphery, analogous to schizophrenia patients (13-16).

In the last decade, a growing number of studies in $\mathrm{BD}$ has been published and TRYCATs are represented as promising biomarkers related to BD (17). However, studies show conflicting results and there is a great variation in methodological quality between studies, with a potential risk of bias as a consequence. Two previous meta-analyses synthesized the role of kynurenine metabolites in broad psychiatric disorders $(18,19)$. Both included a limited number of studies in BD which investigated only a limited selection of TRYCATs (mostly TRP, $\mathrm{KYN}$ and/or KA) and the impact of mood state was not investigated. Arnone and colleagues (18) reported no significant differences in peripheral KYN or TRP values compared to controls, but only five studies were included and there was considerable heterogeneity among studies. The metaanalysis by Wang and Miller (19). found that CSF levels of KA were significantly increased in euthymic BD patients compared to healthy controls, but this finding were based upon two studies with partly overlapping samples $(15,16)$. A third, recently published, meta-analysis summarized the results of studies on TRYCATs in BD, but they included only studies investigating TRYCAT levels in peripheral blood that were published after 2006. Furthermore, they did not provide a critical evaluation of the study quality (20).

The aim of this meta-analysis is to synthesize the available evidence on peripheral and central TRYCAT alterations in casecontrol studies of $\mathrm{BD}$ patients and to critically evaluate the

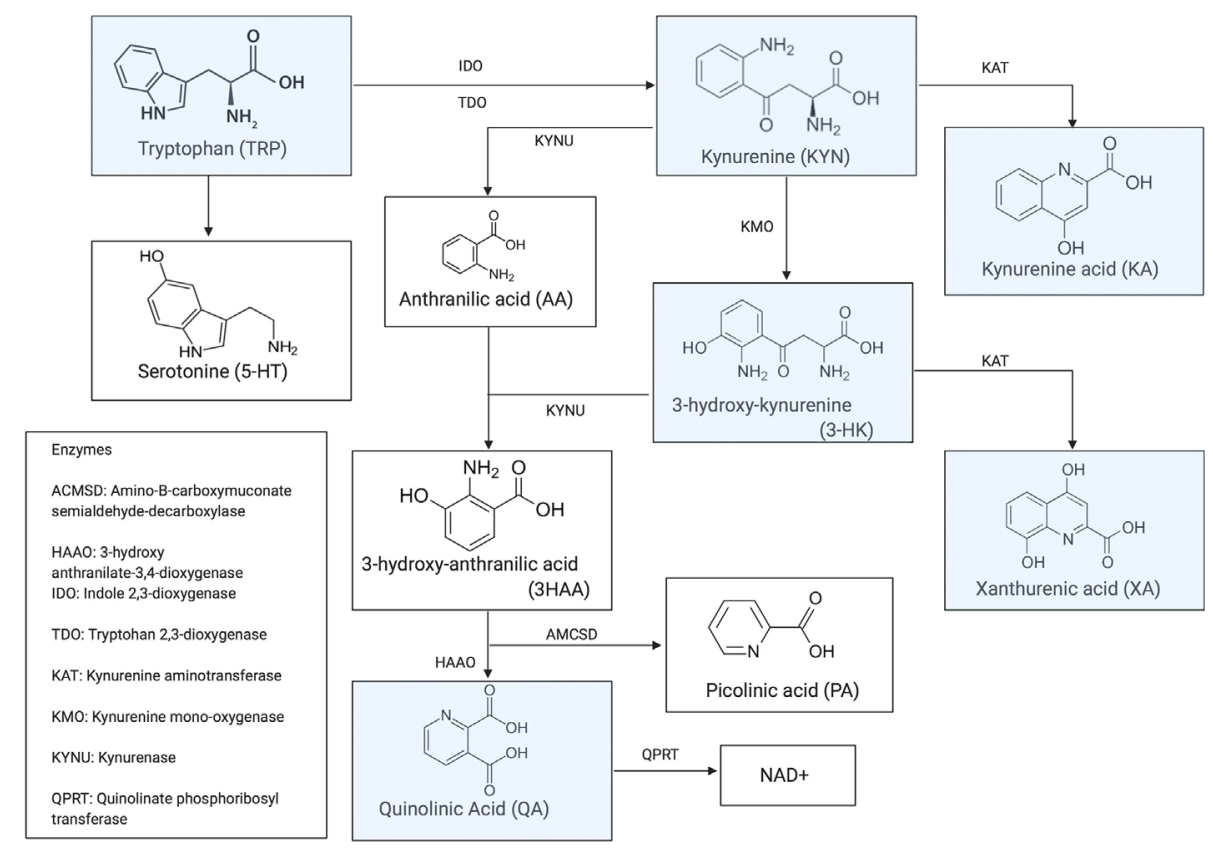

FIGURE 1 | Kynurenine Pathway [previously published in Morrens et al. (4)]. 
quality of available studies. Furthermore, subgroup analyses were performed to separately investigate the differences in TRYCAT levels in manic (BD-M) and bipolar depressed (BD-D) patients.

\section{MATERIAL AND METHODS}

This meta-analysis was conducted and written according to the principles of the PRISMA-P (preferred reporting items for systematic review and meta-analysis protocols) guidelines (21).

\section{Search Strategy}

A search of seven electronic databases (PubMed, Embase, Web of Science, Cochrane, Emcare, PsycINFO, Academic Search Premier) was conducted for original papers on levels of TRYCATs (i.e. TRP, KYN, KA, 3-HK, QA) in BD patients. A medical librarian of the University of Leiden was involved in the establishment of the search strings (see Supplementary S1) and the literature search (last search: August, 19, 2020). Two authors (M.M. and K.H.) independently assessed studies for suitability for inclusion.

Inclusion criteria for eligible papers were: 1) English language papers published in peer-reviewed journals; 2) Case-control studies comparing BD patients (as confirmed by Research Diagnostic Criteria (RDC), DSM-(III, III-R, IV, IV-TR) or ICD-(9 or 10) to healthy controls, 3) assessment of at least 1 TRYCAT metabolite in peripheral blood, CSF or postmortem tissue. In case of sample overlap between studies (as indicated by the authors), only the largest study was included in the current meta-analysis, in order to avoid double counting. Only baseline data were included from longitudinal study articles.

\section{Quality Assessment}

Two researchers (KH and KS) independently assessed the risk of bias and methodological quality of the included studies using a modified version of the Newcastle-Ottawa Quality assessment Scale for case-control studies (22). Following assessments were added to the original scale: an evaluation of the sample size (i.e. a required sample size of minimum twenty patients), assessment of outcome consisting of an evaluation of the completeness of TRYCAT description on the one hand as well as lab procedures (including blinding) in order to guarantee reproducibility on the other hand) and an assessment of statistical reporting. Studies could obtain up to ten stars on three overall quality domains (i.e. selection, comparability, and outcome).

\section{Data Synthesis and Analysis}

Demographic variables (age and gender), clinical assessments (mood state and symptom severity scores), and TRYCAT metabolite levels (means and standard deviations) were extracted from each study. Authors were contacted for additional information when data could not be extracted from the paper; this was received from four papers $(13,23-25)$. The Review Manager 5.3 (RevMan 5.3) computer program was used for performing the primary meta-analysis and subgroup analyses. The primary outcome measure was the standardized mean difference (SMD) in random effect models, represented in forest plot graphs ( $95 \%$ confidence interval). The presence of heterogeneity was assessed using $\mathrm{Chi}^{2}$ and its magnitude using $\mathrm{I}^{2}$ statistics. Potential effect modification by gender, age, and publication year was investigated by performing metaregression analyses (Knapp-Hartung method, maximum likelihood) (26) in Comprehensive Meta-Analysis version 3 (CMA v3). For analyses with ten or more available studies, funnel plots and Egger's tests were used to assess the presence of publication bias.

Subgroup analyses were performed to investigate the difference in TRYCAT levels for BP patients in a different mood state (depressed, manic). A minimum of three studies per subgroup was required in order to perform a subgroup analysis for each TRYCAT. A depressed state was defined as a major depressive episode as diagnosed by the RDC, DSM-(III, III-R, IV, IV-TR) or ICD-(9 or 10) criteria and/or defined as a minimum threshold of 17 or 18 on the Hamilton Rating Scale for Depression (HRSD-17) or 20 on the HRSD-24 (27). A manic state was defined as fulfilling the criteria of the RDC, DSM-(III, III-R, IV, IV-TR) or ICD-(9 or 10) ICD-10) criteria and/or as having a minimum threshold of 13 on the Young Mania Rating Scale (YMRS) (28), the most frequently used scale for assessment of manic symptoms. By means of a supplementary analysis, subgroup analyses were also performed to investigate the differences in effect size between high and low quality studies. The significance level was set at $p<0.05$, the Benjamin-Hochberg procedure was applied for controlling false discovery rates (FDR) in meta-regression analyses.

\section{RESULTS}

\section{Study Selection}

The search strategy resulted in 903 hits and after deduplication 438 remained that were screened for relevance based on title and abstract. A final of 47 papers were read in full, of which 26 were excluded. The PRISMA Flow Diagram in Figure 2 depicts the number of in- and excluded articles from each stage of screening. Four studies investigated TRYCATs in CSF (15, 29-31), sixteen in serum or plasma and one both in CSF and serum (13). Only one post-mortem study met inclusion criteria (32), but this study was excluded due to inadequate reporting. Of the twenty-one included papers in the meta-analysis, twelve had a crosssectional design; nine a longitudinal design. Table 1 presents the characteristics of the included studies. The analysis of TRP in CSF and five TRYCATs (TRP, KYN, KA, 3-HK, QA) in peripheral blood were included in the meta-analyses based on the minimal requirement of three studies for each meta-analysis.

Two CSF studies included both BD-D and BD-M patients (29, 30 ), one solely BD-M (31) and one solely euthymic BD patients (15). Eight serum/plasma studies included only BD-D (12, 23, 33, $35-37,41,42)$, one only BD-M (14), one only euthymic BD (39), two both BD-D and BD-M patients $(24,40)$, one study both BD$\mathrm{M}$ and euthymic BD (34) and two studies BD-D, BD-M and 


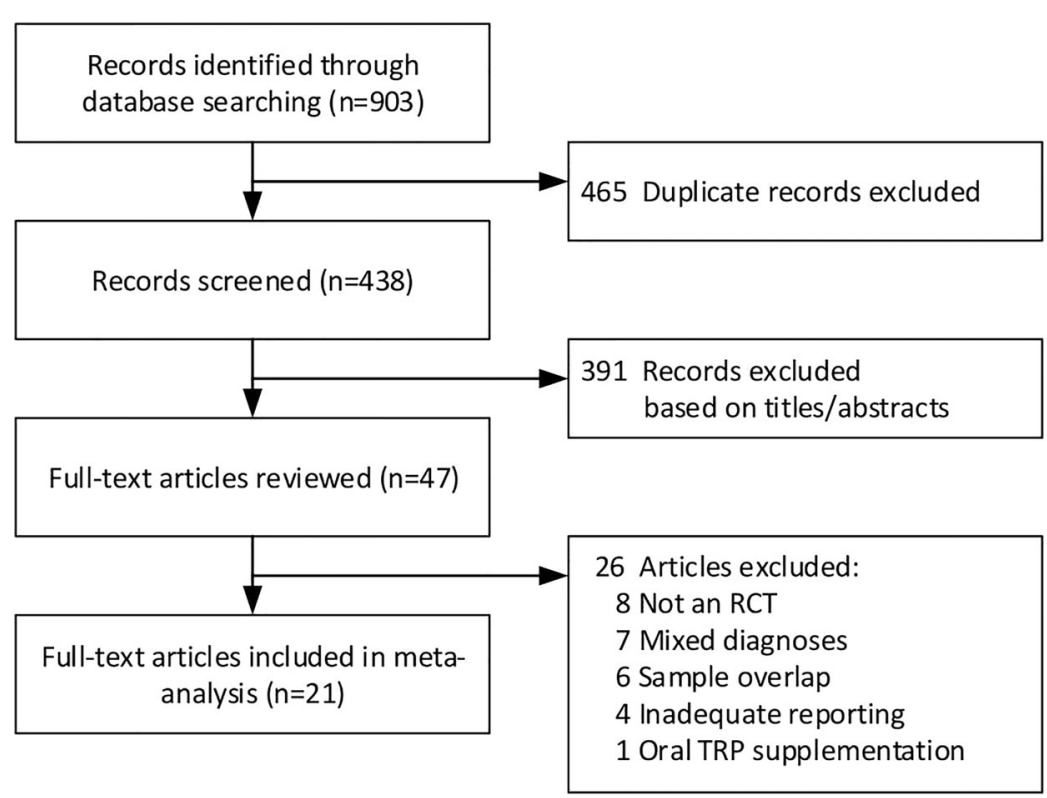

FIGURE 2 | Flowchart.

BD-Mixed patients $(10,17)$. In the two remaining studies the mood state of BD patients was not specified $(13,38)$.

\section{Quality Assessment}

The results of the quality assessment can be found in Table 2 . The quality analysis showed an overall moderate methodological quality with 12 studies (57\%) scoring half of the maximum score or more (5/10 or more). Eight studies (38\%) had a sample size of less than twenty patients $(12,23,29-31,33,36,42)$. Only five studies recruited a matched control sample $(13,23,24,34,38)$ and all but one study (10) reported unadjusted mean TRYCAT levels. Four studies reported that the laboratory technicians were blind for diagnose status $(10,14,31,41)$.

\section{Central Levels of Kynurenine Metabolites}

CSF levels of TRP did not significantly differ from healthy controls $\left(\mathrm{n}_{\text {studies }}=3, \mathrm{n}_{\text {patients }}=39, \mathrm{SMD}=-0.43, z=0.86, p=\right.$ $0.39)$. There was considerable inter-study heterogeneity $\left(\mathrm{I}^{2}: 83 \%\right.$, see Supplementary Figure 4). Only two studies investigated KA in CSF in BD. No CSF studies were found for KYN, 3-HK and $\mathrm{QA}$ in BD. Consequently, these four TRYCATs were not included in the meta-analysis.

\section{Peripheral Levels of Kynurenine Metabolites}

Peripheral blood levels of TRP, KYN and KA were significantly lower in BD compared to healthy controls (TRP: $\mathrm{n}_{\text {studies }}=14, \mathrm{n}_{\text {patients }}=552$, $\mathrm{SMD}=-0.44, z=4.94, p<0.001 ; \mathrm{KYN}: \mathrm{n}_{\text {studies }}=12, \mathrm{n}_{\text {patients }}=514$, $\mathrm{SMD}=-0.30, z=3.21, p=0.001 ; \mathrm{KA}: \mathrm{n}_{\text {studies }}=10, \mathrm{n}_{\text {patients }}=$ 522 , SMD $=-0.45, z=3.98, p<.001$ ). Peripheral QA and 3-HK concentrations did not differ significantly between $\mathrm{BD}$ and healthy controls $\left(\mathrm{QA}: \mathrm{n}_{\text {studies }}=4, \mathrm{n}_{\text {patients }}=203, \mathrm{SMD}=-0.31, z=1.37\right.$, $p=0.17 ; 3-\mathrm{HK}: \mathrm{n}_{\text {studies }}=5, \mathrm{n}_{\text {patients }}=273, \mathrm{SMD}=-0.78, z=0.54$, $p=0.59)$. Inter-study heterogeneity was present for all TRYCATs with $\mathrm{I}^{2}$ ranging from 46 to $77 \%$.

\section{Publication Bias}

Funnel plots (of metabolites with a minimum of 10 available studies; TRP, KYN, KA in peripheral blood) are presented in Supplementary Figures 1 to 3 . The funnel plot of KA shows a significant asymmetry, confirmed by the Egger's test (shown in Table 3), which potentially indicates a publication bias in favor of research reporting lower KA levels in $\mathrm{BD}$.

\section{Subgroup Analyses and Meta-Regression}

Subgroup analyses in euthymic patients could not be reliably performed due to the scarcity of such studies, as there were only three studies including euthymic BD patients, of which one presented CSF levels. Subgroup effect by either depressed or manic mood state for TRP and KYN did not show effect modification $\left(\mathrm{Chi}^{2}\right.$ test for subgroup differences were not significant, see Supplementary Figures 11-12). Subgroup analyses for KA, 3-HK and QA could not be performed since the minimum criterion of three studies in each subgroup was not fulfilled. The pooled effect estimate for TRP in the BD-M subgroup was slightly larger than that of the $\mathrm{BD}-\mathrm{D}$ subgroup (BD-M: SMD = - 0.52; $\mathrm{z}=2.32 ; p=0.02 ; \mathrm{BD}-\mathrm{D}: \mathrm{SMD}=-0.43$; $\mathrm{Z}=2.96 ; p=0.003)$. The pooled effect estimates for KYN in BD-D and $\mathrm{BD}-\mathrm{M}$ groups were comparable $(\mathrm{BD}-\mathrm{M}: \mathrm{SMD}=-0.27 ; \mathrm{z}=$ 1.98; $p=0.05 ; \mathrm{BD}-\mathrm{D}: \mathrm{SMD}=-0.38 ; \mathrm{z}=2.8 ; p=0.005)$. Considerate within-subgroup heterogeneity remained, indicating that other unidentified factors likely affect TRYCAT 
TABLE 1 | Characteristics of included studies

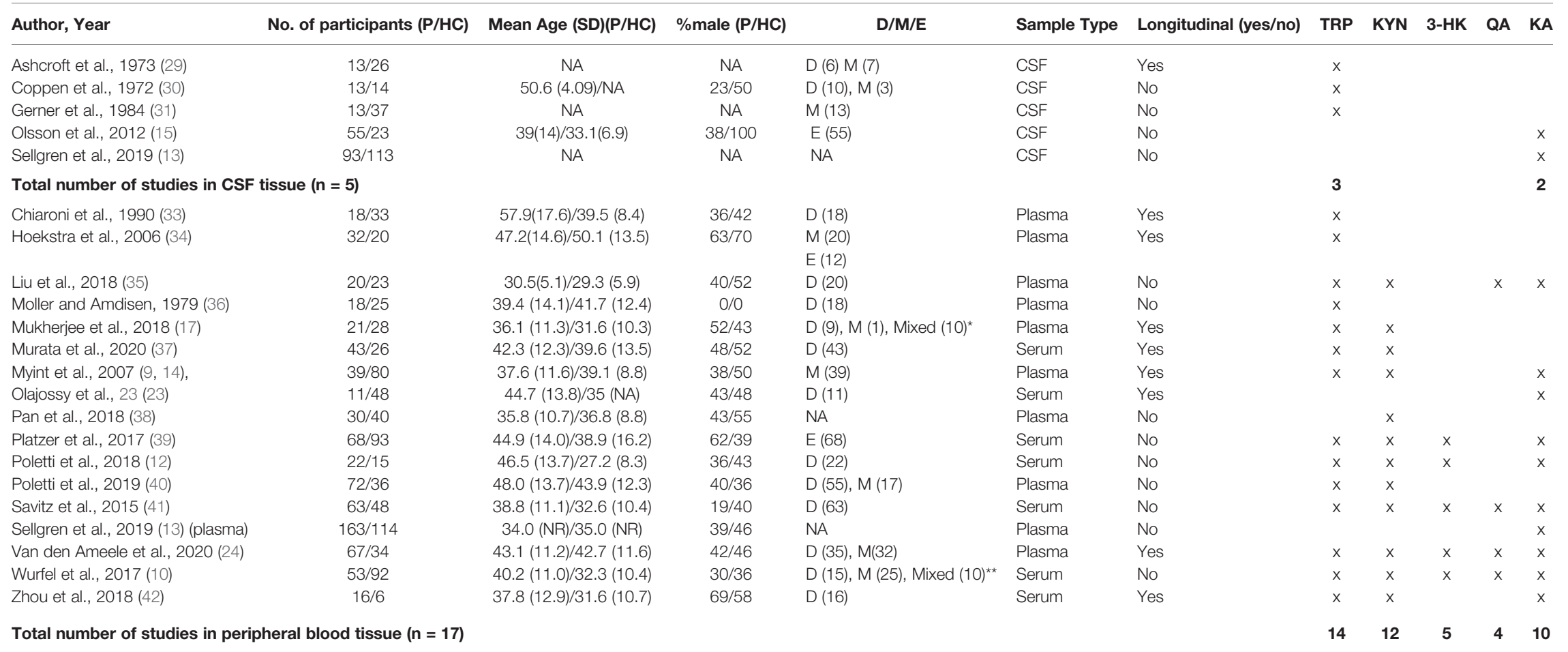

Total number of studies in peripheral blood tissue $(n=17)$

${ }^{*}$ One patient did not have CARS-M data.

SD, standard deviation; P, Patients; HC, healthy controls; D, Depressed; M, Manic; E, Euthymic; TRP, Tryptophan; KYN, kynurenine; 3-HK, 3-hydroxykynurenine; QA, Quinolinic acid; KA, Kynurenic Acid; CSF, cerebrospinal fluid; NA, not applicable. An overview of the total number of studies in CSF and peripheral blood are shown in bold. 
TABLE 2 | Quality Analysis.

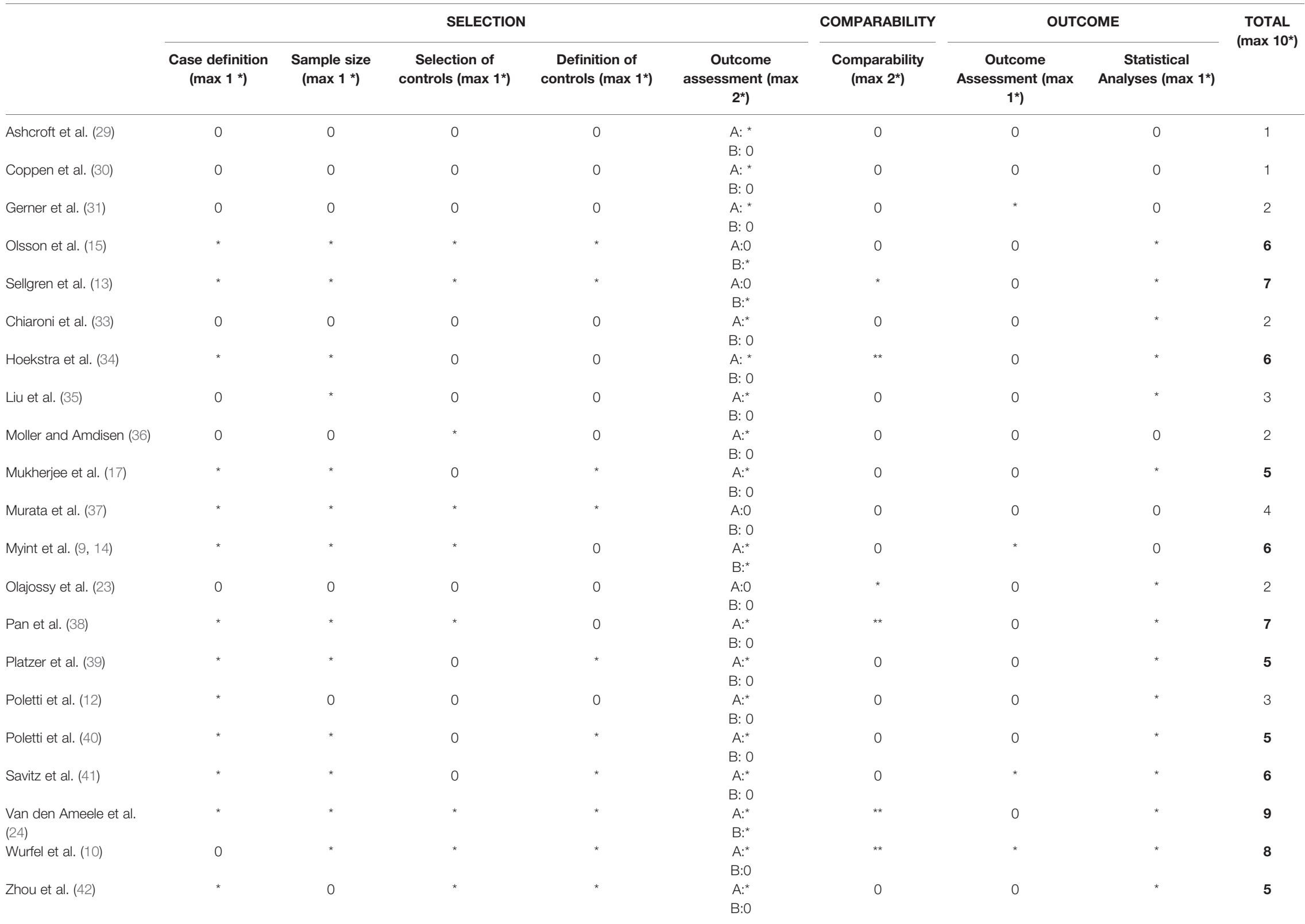

0: not satisfying minimum requirements (see Supplement S1)

* or ${ }^{* *}$ in case of a maximum score of $2^{* *}$ : adequately satisfying minimum requirements. 
TABLE 3 | Results of Egger's tests for publication bias.

\begin{tabular}{lccc}
\hline & Intercept & 95\% C.I. & $\boldsymbol{p ~ ( t w o - t a i l e d ) ~}$ \\
\hline $\mathrm{TRP}_{\text {peripheral }}$ & -1.693 & -4.375 to 0.988 & 0.194 \\
$\mathrm{KYN}_{\text {peripheral }}$ & -1.946 & -4.799 to 0.907 & 0.160 \\
$\mathrm{KA}_{\text {peripheral }}$ & -2.812 & -5.231 to -0.393 & $\mathbf{0 . 0 2 8 ^ { * }}$
\end{tabular}

TRP, tryptophan; KYN, kynurenine; KA, kynurenic acid; C.I., Confidence interval. *significant outcomes are shown in bold.

levels in BD patients. By means of a supplementary analysis (Supplementary Table 1), we performed a subgroup analysis comparing effect sizes in high and low quality studies and this indicated a significant subgroup effect for KYN $(p=0.04)$ and KA $(p=0.04)$ with low quality studies showing larger effect sizes compared to high quality studies.

As demonstrated in the meta-regression analyses (see Supplementary Table 2), there was no effect modification for TRP, KYN and KA by age. The gender of the control group appeared to be a significant moderator of the effect in the studies comparing $\mathrm{KA}$ in $\mathrm{BD}$ and controls, yet this was no longer significant after correcting for false discovery rates. Metaregressions could not be performed for 3-HK and QA due to the low number of studies ( $n=5$ and $n=4$ respectively).

\section{DISCUSSION}

This meta-analysis summarizes the available evidence on a wide range of TRYCAT metabolites, representative for the whole kynurenine pathway, in $\mathrm{BD}$ patients compared to healthy controls. Patients with BD showed lower peripheral levels of TRP, KYN and KA compared to healthy controls. The levels of 3HK and QA were not significantly different between healthy controls and BD. CSF levels of TRP showed no significant difference between $\mathrm{BD}$ and healthy controls, but this finding was based on only three studies.

Our results confirm that $\mathrm{BD}$ is associated with alterations in TRYCATs. However, these findings do not entirely correspond to the theoretically proposed hypotheses to explain the relationship between inflammation, kynurenine metabolism and BD. TRYCATs are assumed to act as inflammatory mediators and to cause neurodegeneration through neurotoxic effects (43), but the exact pathophysiological mechanism how TRYCATS influence BD symptoms and course remain unclear. The lower TRP levels in peripheral blood are consistent with the inefficient serotonin turnover in $\mathrm{BD}(14,17)$, but our findings are not consistent with the theoretical hypothesis of an increased TRP breakdown, under low-grade inflammatory conditions (11), which would be expected to result in elevated KYN and KA levels. A plausible explanation for this inconsistency may be that a proposed microglial branch upregulation results in a reduced shunt towards the astrocytic branch, resulting in lower KYNA levels (44).

Our findings are in line with a recent meta-analysis by our group on TRYCAT alterations in schizophrenia spectrum disorder (SSD) which showed a partial downregulation of the kynurenine pathway (significantly lower levels of peripheral TRP in all SSD patients but especially in acute psychotic, younger patients and of peripheral KA and QA in symptomatic and/or older SSD patients (4). Accumulating evidence shows that acute psychotic exacerbations are associated with different immunological alterations than non-acute states $(45,46)$ and our group previously hypothesized differences in state (i.e. emerging during acute exacerbations) and trait immune markers (i.e. relatively unaltered throughout the disorder) in SSD (47), which could also be the case in BD.

However, it should be noted that peripheral, rather than central TRYCAT metabolites have been measured in most studies. An important question is to what extent CSF and plasma TRYCAT levels are correlated and how they differentially influence the pathophysiology of BD. TRP, KYN, and 3-HK easily cross the blood brain barrier by active transport, but the brain uptake of QA and KA is limited to passive diffusion due to their polarity (48). Sellgren et al. (13) have demonstrated that peripheral KA levels do not mirror central levels in a large sample of BD and healthy controls. But other studies did show a correlation between QA and KA levels in serum and CSF in depressed patients with proven signs of inflammation levels (49, 50). A secondary issue concerns the binding capacity of TRP, KYN and KA to plasma proteins, such as albumin, but the exact result on peripheral values and blood-brain transport remains unclear (48). Third, the peripheral kynurenine pathway is regulated by immune markers, steroids and growth factors (51-53) which can also potentially affect peripheral levels.

All analyses of studies investigating the TRYCAT levels in peripheral blood showed substantial between-study heterogeneity, with effect sizes varying noticeably between studies. This suggests that a number of confounders and study-specific variables contribute to the effect size and, consequently, to the divergence in study results. We investigated the role of mood state (manic or depressed state) in subgroup analyses but this did not explain a significant proportion of the between-study variance. In a further attempt to reveal study-specific characteristics related with heterogeneity, meta-regression analyses were performed but these revealed no significant associations between TRYCAT levels and variables such as age, gender and publication year. It should be emphasized that other, not-investigated, factors could play a role in this heterogeneity. We can broadly categorize these factors into three domains: methodological, clinical and conceptual issues. Apart from differences in methodological quality between studies, differences in lab techniques could also lead to heterogeneous results. Although Liquid-Chromatography Mass Spectrometry is currently considered as golden standard and consequently the most commonly used method, other techniques have been used in studies such as High-Pressure Liquid Chromatography and Atomic Absorption Spectrophotometry). Moreover, some TRYCAT metabolites (such as QA) have extremely low concentrations in peripheral blood tissue which tend to border the limits of the detection range of most of these methods, which may greatly affect reliability of some of these assessments. Several clinical factors are assumed to influence TRYCAT levels, the most of which is the use of psychotropic drugs. Several studies have demonstrated a 
moderating effect of anticonvulsants (e.g. valproate) on TRYCAT levels $(24,34)$ but there is a lack of large-scale studies. Moreover, age and duration of illness may similarly have an effect on TRYCAT changes, although the limited amount of available studies do not allow for proper analyses of these effects. Lastly, between-study heterogeneity could be a reflection of underlying genetic, phenotypical or diagnostic diversity of $\mathrm{BD}$ patients included in different studies (54). However, this heterogeneity, which may translate in differential impact on the TRYCAT pathway, has never been investigated in $\mathrm{BD}$ patient groups.

To our knowledge, this meta-analysis provides the most extensive summary of all available studies on a wide range of TRYCAT levels measured in CSF or serum/plasma in BD patients published to date. Compared to previously published metaanalyses $(18,19)$, we performed a broader literature search and provided a more complete analysis of the data by contacting authors for additional data on TRYCAT levels of BD subgroups. Other strengths of our study are the critical quality assessment of the included studies and the separate analysis of TRYCAT alterations in $\mathrm{BD}$ patients in a different mood (manic, depressed) resulting in a more nuanced picture of TRYCAT alterations in $\mathrm{BD}$ and adding evidence to the discussion on whether TRYCAT alterations should be considered as state or trait dependent changes. However, our results need to be interpreted in view of some limitations. Some analyses included only a small number of studies and the methodological quality of some studies was insufficient. The interpretation of our results is further limited by the differential use of psychopharmacological treatments between patients within and between studies as these are known to have a confounding influence on inflammatory mediators. The majority of the individual studies did not adjust the analysis for important confounders, such as age, gender, smoking status, duration of $\mathrm{BD}$, (doses of) psychotropics, and symptom severity.

\section{REFERENCES}

1. Grande I, Berk M, Birmaher B, Vieta E. Bipolar Disorder. Lancet (2016) 387:1561-72. doi: 10.1016/S0140-67361500241-X

2. Drexhage RC, Hoogenboezem TH, Versnel MA, Berghout A, Nolen WA, Drexhage HA. The Activation of Monocyte and T Cell Networks in Patients With Bipolar Disorder. Brain Behav Immun (2011) 25:1206-13. doi: 10.1016/ j.bbi.2011.03.013

3. Heisler JM, O'Connor JC. Indoleamine 2,3-Dioxygenase-Dependent Neurotoxic Kynurenine Metabolism Mediates Inflammation-Induced Deficit in Recognition Memory. Brain Behav Immun (2015) 50:115-24. doi: 10.1016/j.bbi.2015.06.022

4. Morrens M, De Picker L, Kampen JK, Coppens V. Blood-Based Kynurenine Pathway Alterations in Schizophrenia Spectrum Disorders: A Meta-Analysis. Schizophr Res (2020) 223:43-52. doi: 10.1016/j.schres.2020.09.007

5. Dantzer R. Role of the Kynurenine Metabolism Pathway in InflammationInduced Depression: Preclinical Approaches. Curr Top Behav Neurosci (2017) 31:117-38. doi: 10.1007/7854_2016_6

6. Widner B, Laich A, Sperner-Unterweger B, Ledochowski M, Fuchs D. Neopterin Production, Tryptophan Degradation, and Mental Depression What is the Link? Brain Behav Immun (2002) 16(5):590-5. doi: 10.1016/ S0889-15910200006-5

7. Myint AM, Kim YK. Network Beyond IDO in Psychiatric Disorders: Revisiting Neurodegeneration Hypothesis. Prog Neuropsychopharmacol Biol Psychiatry (2014) 48:304-13. doi: 10.1016/j.pnpbp.2013.08.008

\section{RECOMMENDATIONS FOR FURTHER RESEARCH}

Peripheral TRYCAT levels were lower in BD than healthy controls, signaling a potential role in its pathophysiology. Our results indicate an overall lack of well-powered studies measuring downstream TRYCATs in BD. Future studies should aim to investigate intraindividual analyses of both peripheral and central TRYCAT levels, preferably in a longitudinal design, including patient groups stratified in symptomatic, medicated and age groups.

\section{DATA AVAILABILITY STATEMENT}

The original contributions presented in the study are included in the article/Supplementary Material. Further inquiries can be directed to the corresponding author.

\section{AUTHOR CONTRIBUTIONS}

$\mathrm{KH}$ and $\mathrm{MM}$ performed the literature search and statistical analyses. EG verified the analytical methods. $\mathrm{KH}$ and $\mathrm{KS}$ performed the quality analysis. All authors discussed the results and contributed to the final manuscript. All authors contributed to the article and approved the submitted version.

\section{SUPPLEMENTARY MATERIAL}

The Supplementary Material for this article can be found online at: https://www.frontiersin.org/articles/10.3389/fimmu.2021. 667179/full\#supplementary-material

8. Savitz J. Role of Kynurenine Metabolism Pathway Activation in Major Depressive Disorders. Curr Top Behav Neurosci (2017) 31:249-68. doi: 10.1007/7854_2016_12

9. Myint AM, Kim YK, Verkerk R, Scharpé S, Steinbusch H, Leonard B. Kynurenine Pathway in Major Depression: Evidence of Impaired Neuroprotection. J Affect Disord (2007b) 98:143-51. doi: 10.1016/j.jad.2006. 07.013

10. Wurfel BE, Drevets WC, Bliss SA, McMillin JR, Suzuki H, Ford BN, et al. Serum Kynurenic Acid is Reduced in Affective Psychosis. Transl Psychiatry (2017) 7(5):e1115. doi: 10.1038/tp.2017.88

11. Birner A, Platzer M, Bengesser SA, Dalkner N, Fellendorf FT, Queissner R, et al. Increased Breakdown of Kynurenine Towards its Neurotoxic Branch in Bipolar Disorder. PLoS One (2017) 12:1-14. doi: 10.1371/journal. pone.0172699

12. Poletti S, Myint AM, Schüetze G, Bollettini I, Mazza E, Grillitsch D, et al. Kynurenine Pathway and White Matter Microstructure in Bipolar Disorder Eur Arch Psychiatry Clin Neurosci (2018) 268:157-68. doi: 10.1007/s00406016-0731-4

13. Sellgren CM, Gracias J, Jungholm O, Perlis RH, Engberg G, Schwieler L, et al. Peripheral and Central Levels of Kynurenic Acid in Bipolar Disorder Subjects and Healthy Controls. Transl Psychiatry (2019) 9(1):37. doi: 10.1038/s41398019-0378-9

14. Myint AM, Kim YK, Verkerk R, Park SH, Scharpé S, Steinbusch HWM, et al. Tryptophan Breakdown Pathway in Bipolar Mania. J Affect Disord (2007a) 102:65-72. doi: 10.1016/j.jad.2006.12.008 
15. Olsson SK, Sellgren C, Engberg G, Landén M, Erhardt S. Cerebrospinal Fluid Kynurenic Acid is Associated With Manic and Psychotic Features in Patients With Bipolar I Disorder. Bipolar Disord (2012) 14:719-26. doi: 10.1111/ bdi. 12009

16. Olsson SK, Samuelsson M, Saetre P, Lindström L, Jönsson EG, Nordin C, et al. Elevated Levels of Kynurenic Acid in the Cerebrospinal Fluid of Patients With Bipolar Disorder. J Psychiatry Neurosci (2010) 35:195-9. doi: 10.1503/ jpn.090180

17. Mukherjee D, Krishnamurthy VB, Millett CE, Reider A, Can A, Groer M, et al. Total Sleep Time and Kynurenine Metabolism Associated With Mood Symptom Severity in Bipolar Disorder. Bipolar Disord (2018) 20:27-34. doi: 10.1111/bdi.12529

18. Arnone D, Saraykar S, Salem H, Teixeira AL, Dantzer R, Selvaraj S. Role of Kynurenine Pathway and its Metabolites in Mood Disorders: A Systematic Review and Meta-Analysis of Clinical Studies. Neurosci Biobehav Rev (2018) 92:477-85. doi: 10.1016/j.neubiorev.2018.05.031

19. Wang AK, Miller BJ. Meta-Analysis of Cerebrospinal Fluid Cytokine and Tryptophan Catabolite Alterations in Psychiatric Patients: Comparisons Between Schizophrenia, Bipolar Disorder, and Depression. Schizophr Bull (2018) 44:75-83. doi: 10.1093/schbul/sbx035

20. Bartoli F, Misiak B, Callovini T, Cavaleri D, Cioni RM, Crocamo C, et al. The Kynurenine Pathway in Bipolar Disorder: A Meta-Analysis on the Peripheral Blood Levels of Tryptophan and Related Metabolites. Mol Psychiatry (2020). doi: 10.1038/s41380-020-00913-1

21. Moher D, Shamseer L, Clarke M, Ghersi D, Liberati A, Petticrew M, et al. Preferred Reporting Items for Systematic Review and Meta-Analysis Protocols PRISMA-P 2015 Statement. Rev Esp Nutr Humana y Diet (2016) 20:148-60. doi: 10.1186/2046-4053-4-1

22. Peterson J, Welch V, Losos M, Shea B, O' Connell D, Tugwell P, et al. The Newcastle-Ottawa Scale NOS for Assessing the Quality of Nonrandomised Studies in Meta-Analyses. Ottawa: Ottawa Hospital Research Institute (2011).

23. Olajossy M, Olajossy B, Wnuk S, Potembska E, Urbańska E. Blood Serum Concentrations of Kynurenic Acid in Patients Diagnosed With Recurrent Depressive Disorder, Depression in Bipolar Disorder, and Schizoaffective Disorder Treated With Electroconvulsive Therapy. Psychiatr Pol (2017) 51:455-68. doi: 10.12740/pp/61584

24. van den Ameele S, van Nuijs AL, Lai FY, Schuermans J, Verkerk R, van Diermen L, et al. A Mood State-Specific Interaction Between Kynurenine Metabolism and Inflammation is Present in Bipolar Disorder. Bipolar Disord (2020) 22:59-69. doi: 10.1111/bdi.12814

25. Zhou Y, Zheng W, Liu W, Wang C, Zhan Y, Li H, et al. Cross-Sectional Relationship Between Kynurenine Pathway Metabolites and Cognitive Function in Major Depressive Disorder. Psychoneuroendocrinology (2019) 101:72-9. doi: 10.1016/j.psyneuen.2018.11.001

26. Tipton E, Pustejovsky JE, Ahmadi H. Current Practices in Meta-Regression in Psychology, Education, and Medicine. Res Synth Methods (2019) 10:180-94. doi: $10.1002 /$ jrsm. 1339

27. Hamilton M. A Rating Scale for Depression. J Neurol Neurosurg Psychiatry (1960) 23:56-62. doi: 10.1136/JNNP.23.1.56

28. Young RC, Biggs JT, Ziegler VE, Meyer DA. A Rating Scale for Mania: Reliability,Validity and Sensitivity. Br J Psychiatry J Ment Sci (1978) 133:42935. doi: 10.1192/bjp.133.5.429

29. Ashcroft GW, Blackburn IM, Eccleston D, Glen AI, Hartley W, Kinloch NE, et al. Changes on Recovery in the Concentrations of Tryptophan and the Biogenic Amine Metabolites in the Cerebrospinal Fluid of Patients With Affective Illness. Psychol Med (1973) 3:319-25. doi: 10.1017/s00332917 00049606

30. Coppen A, Brooksbank BW, Peet M. Tryptophan Concentration in the Cerebrospinal Fluid of Depressive Patients. Lancet (1972) 1:1393. doi: 10.1016/s0140-67367291123-3

31. Gerner RH, Fairbanks L, Anderson GM, Young JG, Scheinin M, Linnoila M, et al. CSF Neurochemistry in Depressed, Manic, and Schizophrenic Patients Compared With That of Normal Controls. Am J Psychiatry (1984) 141:153340. doi: 10.1176/ajp.141.12.1533

32. Miller CL, Llenos IC, Cwik M, Walkup J, Weis S. Alterations in Kynurenine Precursor and Product Levels in Schizophrenia and Bipolar Disorder. Neurochem Int (2008) 52:1297-303. doi: 10.1016/j.neuint.2008.01.013
33. Chiaroni P, Azorin JM, Bovier P, Widmer J, Jeanningros R, Barré A, et al. A Multivariate Analysis of Red Blood Cell Membrane Transports and Plasma Levels of L-Tyrosine and L-Tryptophan in Depressed Patients Before Treatment and After Clinical Improvement. Neuropsychobiology (1990) 23:1-7. doi: 10.1159/000118707

34. Hoekstra R, Fekkes D, Loonen AJ, Pepplinkhuizen L, Tuinier S, Verhoeven WM. Bipolar Mania and Plasma Amino Acids: Increased Levels of Glycine. Eur Neuropsychopharmacol (2006) 16:71-7. doi: 10.1016/j.euroneuro.2005. 06.003

35. Liu H, Ding L, Zhang H, Mellor D, Wu H, Zhao D, et al. The Metabolic Factor Kynurenic Acid of Kynurenine Pathway Predicts Major Depressive Disorder. Front Psychiatry (2018) 9:552. doi: 10.3389/fpsyt.2018.00552

36. Moller SE, Amdisen A. Plasma Neutral Amino Acids in Mania and Depression: Variation During Acute and Prolonged Treatment With LTryptophan. Biol Psychiatry (1979) 14:131-9.

37. Murata S, Murphy M, Hoppensteadt D, Fareed J, Welborn A, Halaris A. Effects of Adjunctive Inflammatory Modulation on IL-1 $\beta$ in Treatment Resistant Bipolar Depression. Brain Behav Immun (2020) 87:369-76. doi: 10.1016/j.bbi.2020.01.004

38. Pan JX, Xia JJ, Deng FL, Liang WW, Wu J, Yin BM, et al. Diagnosis of Major Depressive Disorder Based on Changes in Multiple Plasma Neurotransmitters: A Targeted Metabolomics Study. Transl Psychiatry (2018) 8:130. doi: 10.1038/s41398-018-0183-x

39. Platzer M, Dalkner N, Fellendorf FT, Birner A, Bengesser SA, Queissner R, et al. Tryptophan Breakdown and Cognition in Bipolar Disorder. Psychoneuroendocrinology (2017) 81:144-50. doi: 10.1016/j.psyneuen. 2017.04.015

40. Poletti S, Melloni E, Aggio V, Colombo C, Valtorta F, Benedetti F, et al. Grey and White Matter Structure Associates With the Activation of the Tryptophan to Kynurenine Pathway in Bipolar Disorder. J Affect Disord (2019) 259:40412. doi: 10.1016/j.jad.2019.08.034

41. Savitz J, Dantzer R, Wurfel BE, Victor TA, Ford BN, Bodurka J, et al. Neuroprotective Kynurenine Metabolite Indices are Abnormally Reduced and Positively Associated With Hippocampal and Amygdalar Volume in Bipolar Disorder. Psychoneuroendocrinology (2015) 52:200-11. doi: 10.1016/ j.psyneuen.2014.11.015

42. Zhou Y, Zheng W, Liu W, Wang C, Zhan Y, Li H, et al. Antidepressant Effect of Repeated Ketamine Administration on Kynurenine Pathway Metabolites in Patients With Unipolar and Bipolar Depression. Brain Behav Immun (2018) 74:205-12. doi: 10.1016/j.bbi.2018.09.007

43. Anderson G, Maes M. Metabolic Syndrome, Alzheimer Disease, Schizophrenia, and Depression: Role for Leptin, Melatonin, Kynurenine Pathways, and Neuropeptides. In: Faroqui A, Faroqui T, editors. M., Syndome and Neurolgical Disorders. Wiley (2013). p. 235-248.

44. Garrison AM, Parrott JM, Tuñon A, Delgado J, Redus L, O'Connor JC Kynurenine Pathway Metabolic Balance Influences Microglia Activity: Targeting Kynurenine Monooxygenase to Dampen Neuroinflammation. Psychoneuroendocrinology (2018) 94:1-10. doi: 10.1016/j.psyneuen. 2018.04.019

45. De Picker LJ, Morrens M, Chance SA, Boche D. Microglia and Brain Plasticity in Acute Psychosis and Schizophrenia Illness Course: A Meta-Review. Front Psychiatry (2017) 16(8):238. doi: 10.3389/fpsyt.2017.00238

46. Miller BJ, Buckley P, Seabolt W, Mellor A, Kirkpatrick B. Meta-Analysis of Cytokine Alterations in Schizophrenia: Clinical Status and Antipsychotic Effects. Biol Psychiatry (2011) 70:663-71. doi: 10.1016/j.biopsych. 2011.04.013

47. De Picker L, Fransen E, Coppens V, Timmers M, de Boer P, Oberacher H, et al. Immune and Neuroendocrine Trait and State Markers in Psychotic Illness: Decreased Kynurenines Marking Psychotic Exacerbations. Front Immunol (2020) 10:2971. doi: 10.3389/fimmu.2019.02971

48. Fukui S, Schwarcz R, Rapoport SI, Takada Y, Smith QR. Blood-Brain Barrier Transport of Kynurenines: Implications for Brain Synthesis and Metabolism. J Neurochem (1991) 56:2007-17. doi: 10.1111/j.1471-4159.1991.tb03460.x

49. Bay-Richter C, Linderholm KR, Lim CK, Samuelsson M, Träskman-Bendz L, Guillemin GJ, et al. A Role for Inflammatory Metabolites as Modulators of the Glutamate N-Methyl-D-Aspartate Receptor in Depression and Suicidality. Brain Behav Immun (2015) 43:110-7. doi: 10.1016/j.bbi. 2014.07.012 
50. Raison CL, Dantzer R, Kelley KW, Lawson MA, Woolwine BJ, Vogt G, et al. CSF Concentrations of Brain Tryptophan and Kynurenines During Immune Stimulation With IFN- $\alpha$ : Relationship to CNS Immune Responses and Depression. Mol Psychiatry (2010) 15:393-403. doi: 10.1038/ mp.2009.116

51. Belladonna ML, Grohmann U, Guidetti P, Volpi C, Bianchi R, Fioretti MC, et al. Kynurenine Pathway Enzymes in Dendritic Cells Initiate Tolerogenesis in the Absence of Functional IDO. J Immunol (2006) 177:130-7. doi: 10.4049/ jimmunol.177.1.130

52. Huang L, Baban B, Johnson BA, Mellor AL. Dendritic Cells, Indoleamine 2,3 Dioxygenase and Acquired Immune Privilege. Int Rev Immunol (2010) 29 (2):133-55. doi: 10.3109/08830180903349669

53. Salter M, Pogson CI. The Role of Tryptophan 2,3-Dioxygenase in the Hormonal Control of Tryptophan Metabolism in Isolated Rat Liver Cells: Effects of Glucocorticoids and Experimental Diabetes. Biochem J (1985) 229:499-504. doi: 10.1042/bj2290499
54. Askland K, Parsons M. Toward a Biaxial Model of "Bipolar" Affective Disorders: Spectrum Phenotypes as the Products of Neuroelectrical and Neurochemical Alterations. J Affect Disord (2006) 94(1-3):15-33. doi: 10.1016/j.jad.2006.02.024

Conflict of Interest: The authors declare that the research was conducted in the absence of any commercial or financial relationships that could be construed as a potential conflict of interest.

Copyright (c) 2021 Hebbrecht, Skorobogatov, Giltay, Coppens, De Picker and Morrens. This is an open-access article distributed under the terms of the Creative Commons Attribution License (CC BY). The use, distribution or reproduction in other forums is permitted, provided the original author(s) and the copyright owner(s) are credited and that the original publication in this journal is cited, in accordance with accepted academic practice. No use, distribution or reproduction is permitted which does not comply with these terms. 